\title{
NY-ESO-1 Protein Vaccine Plus Montanide ISA-51 VG
}

National Cancer Institute

\section{Source}

National Cancer Institute. NY-ESO-1 Protein Vaccine Plus Montanide ISA-51 VG. NCI

Thesaurus. Code C37507.

A cancer vaccine consisting of an immunogenic peptide derived from the cancer-testis antigen (NY-ESO-1) and emulsified in the immunoadjuvant Montanide ISA-51 VG, with potential immunomodulating and antineoplastic activities. Upon subcutaneous vaccination, the NY-ESO-1 protein vaccine emulsified in Montanide ISA-51 VG may stimulate the host immune system to mount a humoral and cytotoxic T lymphocyte $(C T L)$ response against tumor cells expressing the NY-ESO-1 antigen, resulting in tumor cell lysis. NY-ESO-1, an antigen found in normal testes and various tumors, including bladder, breast, hepatocellular, melanoma, and prostate cancers. The surfactant mannide monooleate in Montanide ISA 51 VG is derived from vegetable-grade (VG) oleic acid that was purified from olive oil. 